\title{
Conservation of energy and momenta in nonholonomic systems with affine constraints*
}

\author{
Francesco FAssò $^{\dagger}$ AND Nicola SANSONETto S $^{\ddagger}$
}

(May 7, 2015)

\begin{abstract}
We characterize the conditions for the conservation of the energy and of the components of the momentum maps of lifted actions, and of their 'gauge-like' generalizations, in time-independent nonholonomic mechanical systems with affine constraints. These conditions involve geometrical and mechanical properties of the system, and are codified in the so-called reaction-annihilator distribution.
\end{abstract}

Keywords: Nonholonomic mechanical systems, Conservation of energy, Reaction-annihilator distribution, Gauge momenta, Nonholonomic Noether theorem.

MSC: 70F25, 37J60, 37J15, 70E18

\section{Introduction}

In this paper we study the conservation of the energy and of the components of the momentum maps of lifted actions - and of their 'gauge' generalizations introduced in [4] - in time-independent mechanical systems with ideal nonholonomic constraints that are affine functions of the velocities. The conservation of both types of functions is affected by the reaction forces exerted by the nonholonomic constraint, but there is a difference between them. The conservation of energy depends on certain properties of the nonhomogeneous term of the constraint distribution, and thus differs from the case of nonholonomic systems with constraints that are linear functions of the velocities. Instead, the conservation of momenta and gauge momenta is essentially the same as that in the case of linear constraints, which has been extensively studied [6, 24, 18, 15]. Therefore, we will mainly focus on the conservation of energy. (If the Lagrangian is not quadratic kinetic energy minus positional potential energy, then the function we call energy is, properly, the Jacobi integral).

It is well known that the energy is conserved in nonholonomic mechanical systems with constraints that are linear in the velocities [28, 27, and that it is not always conserved if the constraints are affine, or more generally nonlinear, in the velocities. More than specifically in the case of affine constraints, the issue of energy conservation has received extensive consideration in the case of general nonlinear constraints 8 , 11, 30, 25, 23,

\footnotetext{
*This work is part of the research projects Symmetries and integrability of nonholonomic mechanical systems of the University of Padova and PRIN Teorie geometriche e analitiche dei sistemi Hamiltoniani in dimensioni finite e infinite.

†Università di Padova, Dipartimento di Matematica, Via Trieste 63, 35121 Padova, Italy. Email: fasso@math.unipd.it

¥Università di Padova, Dipartimento di Matematica, Via Trieste 63, 35121 Padova, Italy. Email: sanson@math. unipd.it

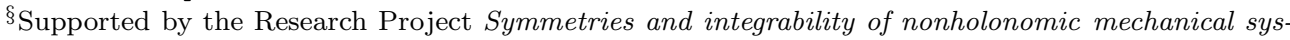
tems of the University of Padova.
} 
10, 5, 21. The equation of balance of the energy shows that the energy is conserved if and only if the reaction forces exerted by the constraint do not do any work on the constrained motions. The quoted references deduce from this some sufficient conditions for the conservation of energy, such as the tangency of the Liouville vector field to the constraint manifold (as e.g. in [25, 11]) or the fact that the constraint is a homogeneous function of the velocities (as e.g. in [10, 5]). However, when particularized to the case of affine constraints, as e.g. in 25, 23, all these sufficient conditions reduce to the linearity of the constraints. It seems, therefore, that conservation of energy for nonholonomic systems with affine constraints is presently not understood. The main purpose of this paper is to remedy this lacune, by identifying the properties of a nonholonomic mechanical system with affine constraints that determine the conservation of its energy.

At the basis of our approach is the fact that, under the hypothesis of ideality of the constraints (namely, d'Alembert principle), the reaction force that the constraint exerts on the system is a known function of the kinematic state of the system. This function depends on properties of the system that are both of geometric nature (the nonholonomic constraint) and of mechanical nature (the mass distribution and the active forces 1 ). The inspection of this function reveals that, for a given system, the set of all reaction forces exerted by the constraints on the constrained motions might be (and typically is) smaller than the set of all reaction forces that satisfy the condition of ideality. The reason is that for a given system the active forces that act on the system are fixed, while the notion of ideality makes reference to all possible active forces that might possibly act on the system (see [19] for a discussion of this fact). Consequently, any vector that annihilates the linear part of the constraint may be an ideal reaction force, but for a given system, the class of reaction forces actually exerted by the constraint may be a subset of this annihilator.

Therefore, the properties of a nonholonomic system that are influenced by the reaction forces exerted on constrained motions may depend, in a complicate way, on the geometric and mechanical properties of the system, including the active forces. These properties can be codified by a distribution on the configuration manifold, which is called the reactionannihilator distribution. This distribution was introduced in 18 in connection with the conservation of the momentum map of lifted actions in nonholonomic systems with linear constraints, and was further used in [15, 16, 19, 13, 17, 22.

We will show that a necessary and sufficient condition for energy conservation in nonholonomic mechanical systems with affine constraints is that the nonhomogeneous term of the constraint is a section of the reaction-annihilator distribution. This clarifies in a quantitative, computable way why energy conservation is not a property of purely geometric type and, in particular, how it depends on the active forces that act on the system: changing just the (conservative) active forces that act on a given system (same constraints, same kinetic energy) may destroy, or restore, the conservation of energy. We will illustrate these behaviours on some examples.

We recall the basic facts about nonholonomic mechanical systems with affine constraints, and introduce the reaction-annihilator distribution for these systems, in Section 2. Energy conservation is studied in Section 3 and the conservation of momenta and gauge momenta of lifted actions is concisely studied in Section 4. A short Conclusion follows, where we stress the importance of exploiting the knowledge of the reaction forces in the study of nonholonomic systems. In the Appendix we derive the expression of the reaction force as function of the kinematic state.

\footnotetext{
${ }^{1} \mathrm{By}$ 'active forces' we mean the forces that act on the system and are not reaction forces; in part of the literature they are called 'external forces'.
} 
Throughout the paper all manifolds and maps are smooth and all vector fields are assumed to be complete. For simplicity we restrict our consideration to time-independent systems. For introductions to nonholonomic mechanics see e.g. [28, 27, 26, 12, 25, 7, 14.

Lastly, we mention that in certain nonholonomic mechanical systems with affine constraints, even if the energy is not conserved, there may exist a modification of it (which may be interpreted as the energy of the system in a moving reference frame and has therefore been called a 'moving energy') that is conserved [20].

\section{Affine constraints and the reaction-annihilator distribution}

2.1. Nonholonomic systems with ideal affine constraints. Since affine constraints appear typically in problems of rigid bodies that roll on moving surfaces, it is appropriate to work on a phase space that is a manifold. However, for simplicity we will resort wherever possible to a coordinate description 2 Moreover, because of the possible presence of moving holonomic constraints in this type of systems, it is natural to allow for the presence of gyrostatic terms in the Lagrangian, that may come either from the use of non-inertial frames or from the use of moving coordinates. We assume however that the system is time-independent, as is it typically happens if the bodies and the surface have suitable symmetries and the latter moves at uniform speed.

Our starting point is thus a Lagrangian system with $n$-dimensional configuration manifold $\hat{Q}$ and Lagrangian $\hat{L}: T \hat{Q} \rightarrow \mathbb{R}$, that describes a mechanical system subject to ideal holonomic constraints. We assume that the Lagrangian has the mechanical form

$$
\hat{L}=\hat{T}-\hat{b}-\hat{V} \circ \pi
$$

where $\hat{T}$ is a positive definite quadratic form on $T \hat{Q}, \hat{b}$ is a 1-form on $\hat{Q}$ regarded as a function on $T \hat{Q}, \hat{V}$ a function on $Q$ and $\pi: T \hat{Q} \rightarrow \hat{Q}$ is the tangent bundle projection. Following e.g. [25] we write Lagrange equations as $[\hat{L}]=0$, where $[\hat{L}]$ may be regarded as a 1 -form on $\hat{Q}$, whose coordinate expression is the well known $\frac{d}{d t} \frac{\partial L}{\partial \dot{q}}-\frac{\partial L}{\partial q}$.

We add now the nonholonomic constraint that, at each point $\hat{q} \in \hat{Q}$, the velocities of the system belong to an affine subspace $\hat{\mathcal{M}}_{\hat{q}}$ of the tangent space $T_{\hat{q}} \hat{Q}$. Specifically, we assume that there are a nonintegrable distribution $\hat{\mathcal{D}}$ on $\hat{Q}$ of constant rank $r$, with $1<r<n$, and a vector field $\hat{\xi}$ on $\hat{Q}$ such that, at each point $\hat{q} \in \hat{Q}$,

$$
\hat{\mathcal{M}}_{\hat{q}}=\hat{\xi}(\hat{q})+\hat{\mathcal{D}}_{\hat{q}}
$$

Clearly, the vector field $\hat{\xi}$ is defined up to a section of $\hat{\mathcal{D}}$. The affine distribution $\hat{\mathcal{M}}$ with fibers $\hat{\mathcal{M}}_{\hat{q}}$ may also be regarded as a submanifold $\hat{M} \subset T \hat{Q}$ of dimension $n+r$, which is actually an affine subbundle of $T \hat{Q}$ of rank $r$, and is called the constraint manifold. The case of linear constraints is recovered when the vector field $\hat{\xi}$ is a section of the distribution $\hat{\mathcal{D}}$, since then $\hat{\mathcal{M}}=\hat{\mathcal{D}}$.

We assume that the nonholonomic constraint is 'ideal', namely, that it satisfies d'Alembert principle. This means that, when the system is in a configuration $\hat{q} \in \hat{Q}$, the set of reaction forces that the nonholonomic constraint is capable of exerting coincides with the annihilator $\hat{\mathcal{D}}_{\hat{q}}^{\circ}$ of $\hat{\mathcal{D}}_{\hat{q}}$ (see e.g. [26, 25]). Under this hypothesis there is a unique function $\hat{R}_{\hat{L}, \hat{M}}: \hat{M} \rightarrow \hat{\mathcal{D}}^{\circ}$, namely a function that associates an ideal reaction force $\hat{R}_{\hat{L}, \hat{M}}$

\footnotetext{
${ }^{2}$ In the sequel, symbols with a hat denote global objects and the same symbols without the hat their local, coordinate representatives.
} 
to each constrained kinematic state $\hat{v}_{\hat{q}} \in \hat{M}$, which has the property that the restriction to $\hat{M}$ of Lagrange equations with the reaction forces,

$$
\left.[\hat{L}]\right|_{\hat{M}}=\hat{R}_{\hat{L}, \hat{M}},
$$

defines a dynamical system on $\hat{M}$ (that is, a vector field on $\hat{M}$ ). For completeness, we give a proof of this fact in the Appendix.

Definition. Assume that $\hat{L}: T \hat{Q} \rightarrow \mathbb{R}$ is as in (1) and that $\hat{M}$ is an affine subbundle of $T \hat{Q}$. The nonholonomic mechanical system with affine constraints $(\hat{L}, \hat{Q}, \hat{M})$ is the dynamical system defined by equation (2) on $\hat{M}$.

2.2. Coordinate description. We consider now a system of local coordinates $q$ on $\hat{Q}$, with domain $Q \subseteq \mathbb{R}^{n}$, and lift them to bundle coordinates $(q, \dot{q}) \in Q \times \mathbb{R}^{n}$ in $T \hat{Q}$. We write the local representative of the Lagrangian $\hat{L}=\hat{T}+\hat{b}-\hat{V} \circ \pi$ as

$$
L(q, \dot{q})=\frac{1}{2} \dot{q} \cdot A(q) \dot{q}+b(q) \cdot q-V(q)
$$

with $A(q)$ an $n \times n$ symmetric nonsingular matrix and $b(q) \in \mathbb{R}^{n}$

The fibers of the local representative $\mathcal{D}$ of the distribution $\hat{\mathcal{D}}$ can be described as the kernel of a $q$-dependent $k \times n$ matrix $S(q)$ that has everywhere rank $k$, with $k=n-r$ :

$$
\mathcal{D}_{q}=\left\{\dot{q} \in T_{q} Q=\mathbb{R}^{n}: S(q) \dot{q}=0\right\} .
$$

The matrix $S$ is not uniquely defined. However, if $S_{1}$ and $S_{2}$ are any two possible choices of it, then from ker $S_{1}=\operatorname{ker} S_{2}$ it follows that there exists a $q$-dependent $k \times k$ nonsingular matrix $P(q)$ such that $S_{2}=P S_{1}$.

Let now $\mathcal{M}$ be the local representative of $\hat{\mathcal{M}}, M \subset Q \times \mathbb{R}^{n}$ that of $\hat{M}$ and $\xi: Q \rightarrow \mathbb{R}^{n}$ that of $\hat{\xi}$. Then $\dot{q} \in \mathcal{M}_{q}$ if and only if $\dot{q}=\xi(q)+u$ for some $u \in \operatorname{ker} S(q)$, that is, if and only if $S(q)[\dot{q}-\xi(q)]=0$. Thus

$$
M=\left\{(q, \dot{q}) \in Q \times \mathbb{R}^{n}: S(q) \dot{q}+s(q)=0\right\}
$$

with

$$
s(q)=-S(q) \xi(q) \in \mathbb{R}^{k} .
$$

Note that $s$ is independent of the arbitrariness in the choice of the component of $\hat{\xi}$ along $\hat{\mathcal{D}}$, that is of the component of $\xi$ along $\operatorname{ker} S$. However, $s$ depends on the choice of the matrix $S$ : if $s_{1}$ and $s_{2}$ are relative to two matrices $S_{1}$ and $S_{2}=P S_{1}$, then $s_{2}=P s_{1}$.

In coordinates, the equations of motion (2) of the nonholonomic mechanical system $(\hat{L}, \hat{Q}, \hat{M})$ are

$$
\left.\left(\frac{d}{d t} \frac{\partial L}{\partial \dot{q}}-\frac{\partial L}{\partial q}\right)\right|_{M}=R_{L, M}
$$

where $R_{L, M}(q, \dot{q})$ is the local representative of $\hat{R}_{\hat{L}, \hat{M}}$. As shown in the Appendix, $R_{L, M}$ equals the restriction to $M$ of the function

$$
S^{T}\left(S A^{-1} S^{T}\right)^{-1}\left(S A^{-1} \ell-\sigma\right)
$$

where $\ell \in \mathbb{R}^{n}$ and $\sigma \in \mathbb{R}^{k}$ have components 3

$$
\ell_{i}=\frac{\partial^{2} L}{\partial \dot{q}_{i} \partial q_{j}} \dot{q}_{j}-\frac{\partial L}{\partial q_{i}}, \quad \sigma_{a}=\frac{\partial S_{a i}}{\partial q_{j}} \dot{q}_{i} \dot{q}_{j}+\frac{\partial s_{a}}{\partial q_{j}} \dot{q}_{j}
$$

\footnotetext{
${ }^{3}$ We use everywhere the convention of summation over repeated indexes.
} 
with $i, j, h=1, \ldots, n$ and $a=1, \ldots, k$. Note that

$$
\ell=\alpha+\beta+V^{\prime}
$$

with $\alpha_{i}=\left(\frac{\partial A_{i j}}{\partial q_{h}}-\frac{1}{2} \frac{\partial A_{j h}}{\partial q_{i}}\right) \dot{q}_{j} \dot{q}_{h}, \beta_{i}=\left(\frac{\partial b_{j}}{\partial q_{i}}-\frac{\partial b_{i}}{\partial q_{j}}\right) \dot{q}_{j}$ and $V_{i}^{\prime}=\frac{\partial V}{\partial q_{i}}$.

In the case of linear constraints, expression (5) or analogue expressions are given in [2, 18, 7]; in the Appendix, besides considering the affine case, we complement these treatments with a global perspective.

Remark. The restriction to $M$ of the function (5) is independent of the choice of $S$ and $s$, that is, under the replacement of $S, s$ by $P S, P s$. (This change produces an extra term in $\sigma$, which however vanishes on $M$ ).

2.3. The reaction-annihilator distribution. While the condition of ideality assumes that, at each point $\hat{q} \in \hat{Q}$, the constraint can-a priori-exert all reaction forces that lie in $\hat{\mathcal{D}}_{\hat{q}}^{\circ}$, expression (5) shows that, ordinarily, only a subset of these possible reaction forces is actually exerted in the motions of the system. In fact, in coordinates, $\hat{\mathcal{D}}_{\hat{q}}^{\circ}$ is the orthogonal complement to ker $S(q)$, namely the range of $S(q)^{T}$, and the map

$$
\left.S^{T}\left(S A^{-1} S^{T}\right)^{-1}\left(S A^{-1} \ell-\sigma\right)\right|_{M_{q}}: M_{q} \rightarrow \operatorname{range} S(q)^{T}
$$

might not be surjective. Specifically, the reaction forces that the constraint exerts, when the system $(\hat{L}, \hat{Q}, \hat{M})$ is in a configuration $\hat{q} \in \hat{Q}$ with any possible velocity $\hat{v}_{\hat{q}} \in \hat{\mathcal{M}}_{\hat{q}}$, are the elements of the set

$$
\hat{\mathcal{R}}_{\hat{q}}:=\bigcup_{\hat{v} \in \hat{\mathcal{M}}_{\hat{q}}} \hat{R}_{\hat{L}, \hat{M}}\left(\hat{v}_{\hat{q}}\right)
$$

and this set may be (and typically is) a proper subset of $\hat{\mathcal{D}}_{\hat{q}}^{\circ}$. This point of view was taken in [18, and leads to the following

Definition. The reaction-annihilator distribution $\hat{\mathcal{R}}^{\circ}$ of a nonholonomic mechanical system with affine constraints $(\hat{L}, \hat{Q}, \hat{M})$ is the (possibly non-smooth and of non-constant rank) distribution on $\hat{Q}$ whose fiber $\hat{\mathcal{R}}_{\hat{q}}^{\circ}$ at $\hat{q} \in \hat{Q}$ is the annihilator of $\hat{\mathcal{R}}_{\hat{q}}$.

The interest of this distribution is not that much geometric, but mechanical: a vector field $\hat{Z}$ on $\hat{Q}$ is a section of $\hat{\mathcal{R}}^{\circ}$ if and only if, in all constrained motions of the system, the reaction force does zero work on it: $\left\langle\hat{R}_{\hat{L}, \hat{M}}\left(\hat{v}_{\hat{q}}\right), \hat{Z}(\hat{q})\right\rangle=0$ for all $\hat{v} \in \hat{M}$. This is a system-dependent condition, which is weaker than being a section of $\hat{\mathcal{D}}$ because

$$
\hat{\mathcal{D}}_{\hat{q}} \subseteq \hat{\mathcal{R}}_{\hat{q}}^{\circ} \quad \forall \hat{q} \in \hat{Q} .
$$

Expression (5) of the reaction forces shows that $\hat{\mathcal{R}}^{\circ}$ is computable a priori, without knowing the motions of the system. Moreover, this expression shows how $\hat{\mathcal{R}}^{\circ}$ depends on the geometry of the nonholonomic constraints (through the matrix $S$ and the vector $s$ ) and on the mass distribution of the system and on the active forces that act on it (through the Lagrangian) 4

Examples of reaction-annihilator distributions $\hat{\mathcal{R}}^{\circ}$, that show that their fibers may actually be larger than those of $\hat{\mathcal{D}}$, are given in [18, 15] for systems with linear constraints and in sections 3.2 and 4.3 below for the case of affine constraints. For a discussion of the relation between $\hat{\mathcal{R}}^{\circ}$ and d'Alembert principle see [19].

${ }^{4} \hat{\mathcal{R}}^{\circ}$ depends of course also on the holonomic constraint, through the dependence of $L$ on $(q, \dot{q})$. 


\section{Conservation of energy in systems with affine constraints}

3.1. Characterization of the conditions for energy conservation. We recall that the energy, or more exactly the Jacobi integral, of a Lagrangian $\hat{L}$ is the function

$$
\hat{E}_{\hat{L}}(\hat{v}):=\left\langle\hat{p}_{\hat{L}}, \hat{v}\right\rangle-\hat{L}(\hat{v})
$$

where $\hat{p}_{\hat{L}}$ is the momentum 1 -form relative to $\hat{L}$, namely $\hat{p}_{\hat{L}}=\mathbf{F} L$ with $\mathbf{F}$ the fiber derivative (as defined, e.g., in [1]). In coordinates, $p_{L}=\frac{\partial L}{\partial \dot{q}}$ and, if $L$ is as in (3), $p_{L}(q, \dot{q})=A(q) \dot{q}-b(q)$.

If $\hat{L}$ is as in (11) then $\hat{E}_{L}=\hat{T}+\hat{V} \circ \pi$. The function $\hat{E}_{\hat{L}}$ can be properly interpreted as the mechanical energy of the system only if $\hat{L}=\hat{T}-\hat{V} \circ \pi$ but, as is customary, we will call it energy in all cases. For a Lagrangian system, $\hat{E}_{\hat{L}}$ is a first integral if and only if, as we do assume here, $\hat{L}$ is independent of time.

Definition. The energy $\hat{E}_{\hat{L}, \hat{M}}$ of the nonholonomic mechanical system with affine constraints $(\hat{L}, \hat{Q}, \hat{M})$ is the restriction of $\hat{E}_{\hat{L}}$ to the constraint manifold $\hat{M}$.

Proposition 1. For a nonholonomic mechanical system with affine constraints $(\hat{L}, \hat{Q}, \hat{M})$ with constraint distribution $\hat{\mathcal{D}}+\hat{\xi}$, the energy $\hat{E}_{\hat{L}, \hat{M}}$ is a first integral if and only if $\hat{\xi}$ is a section of $\hat{\mathcal{R}}^{\circ}$.

Proof. The proof can be given in coordinates. By (4), along any curve $t \mapsto\left(q_{t}, \dot{q}_{t}\right) \in M$,

$$
\frac{d}{d t} E_{L, M}\left(q_{t}, \dot{q}_{t}\right)=\dot{q}_{t} \cdot\left(\frac{d}{d t} \frac{\partial L}{\partial \dot{q}}-\frac{\partial L}{\partial q}\right)\left(q_{t}, \dot{q}_{t}\right)=\dot{q}_{t} \cdot R_{L, M}\left(q_{t}, \dot{q}_{t}\right) .
$$

If $\dot{q} \in \mathcal{M}(q)$, namely $\dot{q}=u+\xi(q)$ with $u \in \mathcal{D}_{q}$, then

$$
R_{L, M}(q, \dot{q}) \cdot \dot{q}=R_{L, M}(q, \dot{q}) \cdot \xi(q)
$$

given that $R_{L, M}$ is ideal and hence annihilates $\mathcal{D}_{q}$. It follows that $E_{L, M}$ is a first integral if and only if $R_{L, M}(q, \dot{q}) \cdot \xi(q)=0$ for all $q \in Q, \dot{q} \in \mathcal{M}_{q}$, that is $\xi(q) \in \mathcal{R}_{q}^{\circ}$ for all $q \in Q$.

This shows that energy conservation is not a universal property of nonholonomic mechanical systems with affine constraints. In particular, as we have already stressed, it depends on the active forces that act on the system.

In this respect we note that at each point $\hat{q} \in \hat{Q}$, the union of the fibers at $\hat{q}$ of the distributions $\hat{\mathcal{R}}^{\circ}$ relative to all functions $\hat{V}: \hat{Q} \rightarrow \mathbb{R}$ equals $\hat{\mathcal{D}}_{q}$. (In fact, in expression (5), $\ell=\alpha+\beta+V^{\prime}$ and the matrix $S^{T}\left(S A^{-1} S^{T}\right)^{-1} S A^{-1}$ is, at each point $q$, the $A(q)^{-1}$-orthogonal projector onto $\mathcal{D}_{q}^{\circ}$ ). Thus, it follows from Proposition 1 that, given $\hat{T}$ and $\hat{M}, \hat{E}_{\hat{L}, \hat{M}}$ is a first integral in all nonholonomic mechanical systems of the class $(\hat{L}=\hat{T}-\hat{V} \circ \pi, \hat{Q}, \hat{M})$, with any $\hat{V}: \hat{Q} \rightarrow \mathbb{R}$, if and only if the constraint is linear. This fact, which can be generalized to allow for the presence of gyrostatic terms in the Lagrangian, is a particular case of a result by [31 for general nonlinear constraints (with linearity replaced by homogeneity).

Remark. The result in Proposition 1 does not depend on the choice of $\xi$, which is defined up to the addition of a section of $\mathcal{D}$, because $\mathcal{D} \subseteq \mathcal{R}^{\circ}$. 
3.2. Examples. 1. An affine nonholonomic particle. In order to illustrate the dependency of energy conservation on the active forces we consider an affine version of Pars' nonholonomic particle [28]. This system has configuration manifold $\mathbb{R}^{3} \ni q=(x, y, z)$, Lagrangian

$$
L(q, \dot{q})=\frac{1}{2}\|\dot{q}\|^{2}-V(q)
$$

with a potential energy $V$ for which we will make different choices, and constraint

$$
\dot{z}+x \dot{y}-y \dot{x}-c=0
$$

with $c$ a nonzero real number. Thus $n=3$ and $k=1, \xi=c \partial_{z}$ and the distribution $\mathcal{D}$ is spanned by the two vector fields $\partial_{x}+y \partial_{z}$ and $x \partial_{x}+y \partial_{y}$. $\mathcal{D}$ has rank 2 except where $y=0$, so we disregard these points and restrict the configuration manifold to $Q=\mathbb{R}^{3} \backslash\{y=0\}$. Note that not only $\xi$ is not a section of $\mathcal{D}$ but, after the restriction to $Q$,

$$
\xi(q) \notin \mathcal{D}_{q} \quad \forall q \in Q
$$

The constraint manifold $M$ is diffeomorphic to $Q \times \mathbb{R}^{2}$ and has global coordinates $(x, y, z, \dot{x}, \dot{y})$. We may take $S(x, y, z)=(-y, x, 1)$ and $s=-c$.

Due to its low dimensionality, this example offers little variety of behaviours. Specifically, the distribution $\mathcal{R}^{\circ}$ depends on the function $V$ but, since its fibers contain those of $\mathcal{D}$, that have dimension 2 , there are only two possibilities: at a point $\bar{q}$, either $\mathcal{R}_{\bar{q}}^{\circ}=T_{\bar{q}} Q=\mathbb{R}^{3}$ or $\mathcal{R}_{\bar{q}}^{\circ}=\mathcal{D}_{\bar{q}}$. The former possibility is realized if $R_{L, M}(\bar{q}, \dot{q})=0$ for all $\dot{q} \in \mathbb{R}^{3}$ and the second if $R_{L, M}(\bar{q}, \dot{q}) \neq 0$ for some $\dot{q} \in \mathbb{R}^{3}$.

At the same time, however, the low dimensionality makes all computations straightforward, and it is simple to find potentials $V$ that exemplify the different possibilities. In fact $A=\mathbb{I}$ and $\alpha=\beta=0$, so $\ell=V^{\prime}$, and $\sigma=0$ and (5) gives

$$
R_{L, M}=\left.S^{T}\left(S S^{T}\right)^{-1} V^{\prime}\right|_{M}=\left.\frac{1}{1+x^{2}+y^{2}}\left(\begin{array}{ccc}
x y^{2} & -x y & -y \\
-x y & x^{2} & x \\
-y & x & 1
\end{array}\right) V^{\prime}\right|_{M}
$$

Thus, the reaction forces are independent of the $\dot{q}$. This would make it straightforward to compute the fibers $\mathcal{R}_{q}^{\circ}$, which are simply the orthogonal complements to $R_{L, M}(q)$ in $\mathbb{R}^{3}$, but we need not doing it because we already know what these fibers are.

Concerning the conservation of energy, there are two cases to consider:

1. If $V$ is such that $R_{L, M}(q)=0$ at all points $q \in Q$, then $\mathcal{R}^{\circ}=\mathbb{R}^{3}$ and energy is conserved 5 From (9) one verifies that this situation is encountered, e.g., with $V=0$ and $V=\frac{1}{2}\left(x^{2}+y^{2}\right)$.

2. If $V$ is such that $R_{L, M}$ is not identically zero, then there is a point $\bar{q} \in Q$ at which $\mathcal{R}_{\bar{q}}^{\circ}=\mathcal{D}_{\bar{q}}$. Thus, by (18),$\xi(\bar{q}) \notin \mathcal{R}_{\bar{q}}^{\circ}$ and so $\xi$ is not a section of $\mathcal{R}^{\circ}$. Hence energy is not conserved. An example is $V=z$.

A richer variety of possibilities, including (a) conservation of energy even with nonzero reaction forces, and (b) violation of the conservation of energy even in the absence of active forces, can be easily constructed by considering four- or five-dimensional extensions of the nonholonomic particle, similar to that considered in [16]. However, cases (a) and (b) are met also in known mechanical systems. For instance, in the system formed by

\footnotetext{
${ }^{5}$ That energy is conserved if the reaction forces vanish identically is of course obvious, for a variety of reasons. For instance, the nonholonomic system is a subsystem of the unconstrained system with Lagrangian $L$ on $T Q$.
} 
a heavy sphere that rolls on a rotating horizontal plane, considered e.g. in 27, the potential energy of the active forces is constant but the energy is not conserved [20]. An example of case (a) is the following one.

2. A sphere rolling inside a rotating cylinder. As a second example we consider the system formed by a homogeneous sphere constrained to roll without sliding inside a cylinder that rotates with constant angular velocity about its figure axis. We assume that the sphere is acted upon by positional forces whose potential energy is a function of the position of the sphere's center of mass. The case of a heavy sphere inside a vertical cylinder that is at rest is classical [29, 27]. The case of rotating cylinder, but without active forces, is considered in [9].

Let $a$ be the radius of the sphere and $r+a$ the radius of the cylinder. The holonomic system we start from is formed by the sphere constrained to keep its center on a cylinder $C$ of radius $r$. Fix an orthonormal frame $\Sigma=\left\{O ; e_{x}, e_{y}, e_{z}\right\}$ with the origin $O$ on the figure axis of the cylinder and $e_{z}$ aligned with it. Using cylindrical coordinates $(z, \gamma)$ relative to $\Sigma$ we identify $C$ with $\mathbb{R} \times S^{1}$ and the configuration manifold is $\hat{Q}=\mathbb{R} \times S^{1} \times \mathrm{SO}(3) \ni$ $(z, \gamma, \mathcal{R})$, where the matrix $\mathcal{R}$ gives the sphere's orientation. Up to an overall factor, the Lagrangian is then

$$
\hat{L}=\frac{1}{2}\left(r^{2} \dot{\gamma}^{2}+\dot{z}^{2}\right)+\frac{I}{2}\|\omega\|^{2}-V(z, \gamma)
$$

where (up to the same factor) $I$ is the moment of inertia of the sphere and $V$ is the potential energy of the active forces, and $\omega=\left(\omega_{x}, \omega_{y}, \omega_{z}\right)$ is the angular velocity of the sphere relative to $\Sigma$ (that we think of as a function on $\operatorname{TSO}(3)$ ). We add now the nonholonomic constraint that the sphere rolls without sliding on a cylinder, coaxial with $C$ and of radius $r+a$, that rotates with constant angular velocity $\Omega e_{z}$ relative to $\Sigma$ :

$$
r \dot{\gamma}+a \omega_{z}-(r+a) \Omega=0, \quad \dot{z}+a\left(\omega_{x} \sin \gamma-\omega_{y} \cos \gamma\right)=0 .
$$

These equations can be solved for $(\dot{z}, \dot{\gamma})$ and the constraint manifold $\hat{M}$ is thus diffeomorphic to $\mathbb{R} \times S^{1} \times T \mathrm{SO}(3)$.

As local coordinates on $\hat{Q}$ we use the cylindrical coordinates $(z, \gamma)$ of the center of mass of the sphere and three Euler angles $(\varphi, \psi, \theta) \in S^{1} \times S^{1} \times(0, \pi)$ that fix the orientation of a body frame relative to $\Sigma$ (we adopt the convention of [3] for the choice of these angles). The representative of $\hat{L}$ is

$$
L=\frac{1}{2}\left(r^{2} \dot{\gamma}^{2}+\dot{z}^{2}\right)+\frac{I}{2}\left(\dot{\theta}^{2}+\dot{\varphi}^{2}+\dot{\psi}^{2}+2 \dot{\varphi} \dot{\psi} \cos \theta\right)-V(z, \gamma)
$$

and the constraint (11) becomes

$$
r \dot{\gamma}+a(\dot{\varphi}+\dot{\psi} \cos \theta)-(r+a) \Omega=0, \quad \dot{z}+a[\dot{\psi} \sin \theta \cos (\gamma-\varphi)+\dot{\theta} \sin (\gamma-\varphi)]=0 .
$$

In these coordinates the vector field $\hat{\xi}$ becomes the constant vector field $\xi=\Omega\left(\partial_{\gamma}+\partial_{\varphi}\right)$, or $\xi=(0, \Omega, \Omega, 0,0)$, and a possible choice of $S$ and $s$ is

$$
S=\left(\begin{array}{ccccc}
0 & r & a & a \cos \theta & 0 \\
1 & 0 & 0 & a \sin \theta \cos (\gamma-\varphi) & a \sin (\gamma-\varphi)
\end{array}\right), \quad s=(0,-(r+a) \Omega) .
$$

As local coordinates on $\hat{M}$ we may use $(z, \gamma, \varphi, \psi, \theta, \dot{\varphi}, \dot{\psi}, \dot{\theta})$. From (15), the reaction force is then

$$
R_{L, M}=\frac{I}{I+a^{2}}\left(f, V_{\gamma}^{\prime}, \frac{a}{r} V_{\gamma}^{\prime}, \text { af } \cos (\gamma-\varphi) \sin \theta+\frac{a}{r} V_{\gamma}^{\prime} \cos \theta, \text { af } \sin (\gamma-\varphi)\right)
$$


with $f=\frac{a^{2}}{r}\left(\dot{\varphi}+\dot{\psi} \cos \theta-\frac{a+r}{a} \Omega\right)(\dot{\theta} \cos (\gamma-\varphi)-\dot{\psi} \sin (\gamma-\varphi) \sin \theta)+V_{z}^{\prime}$. Therefore,

$$
R_{L, M} \cdot \xi=\frac{a+r}{a} \Omega V_{\gamma}^{\prime} .
$$

This shows that, when $\Omega \neq 0$ and hence $\xi \neq 0$ and the constraint is affine, the energy is conserved if and only $V$ depends on $z$ alone. This includes the case of a heavy sphere that rolls inside a rotating vertical cylinder, for which $V=g z$.

\section{Conservation of momenta and gauge momenta of lifted actions}

4.1. Conservation of momenta. We consider now a second problem in which the reaction forces of a nonholonomic mechanical system with affine constraints play a role: the conservation of the momentum map of a lifted action that leaves the Lagrangian $\hat{L}$ invariant, and of its 'gauge' generalization.

This topic has been widely studied in the case of nonholonomic mechanical systems with linear constraints. For such systems, the momentum map is in general not conserved, but in certain cases some of its components are conserved. In early studies, it was pointed out that a sufficient condition for the conservation of a component of the momentum map is that its infinitesimal generator is 'horizontal', that is, a section of the constraint distribution (see e.g. [6, 24, 8]). It was later proved that the components of the momentum map that are conserved are exactly those whose infinitesimal generators are sections of $\mathcal{R}^{\circ}[18$. Our first goal here is to show that this result holds in the case of affine constraints, too. In fact, the affine part of the constraint plays no role in it.

Consider an action $\hat{\Psi}: G \times \hat{Q} \rightarrow \hat{Q}$ of a Lie group $G$ on the configuration manifold $\hat{Q}$. For each $\hat{q} \in \hat{Q}$ we write as usual $\hat{\Psi}_{g}(\hat{q})$ for $\hat{\Psi}(g, \hat{q})$. The tangent lift $\hat{\Psi}^{T \hat{Q}}: G \times T \hat{Q} \rightarrow T \hat{Q}$ of the action $\hat{\Psi}$ is the action of $G$ on $T \hat{Q}$ given by

$$
\hat{\Psi}_{g}^{T \hat{Q}}\left(\hat{v}_{\hat{q}}\right)=T_{\hat{q}} \hat{\Psi}_{g} \cdot \hat{v}_{\hat{q}}
$$

(in coordinates, $\Psi_{g}^{T Q}(q, \dot{q})=\left(\Psi_{g}(q), \Psi_{g}^{\prime}(q) \dot{q}\right)$ with $\left.\Psi_{g}^{\prime}=\frac{\partial \Psi_{g}}{\partial q}\right)$. We denote by $\hat{Y}_{\eta}:=$ $\left.\frac{d}{d t} \hat{\Psi}_{\exp (t \eta)}\right|_{t=0}$ the infinitesimal generator relative to an element $\eta \in \mathfrak{g}$, the Lie algebra of $G$. Correspondingly, the $\eta$-component of the momentum map of $\hat{\Psi}^{T \hat{Q}}$ is the function $\hat{J}_{\eta}: T \hat{Q} \rightarrow \mathbb{R}$ defined as

(in coordinates, $\frac{\partial L}{\partial \dot{q}} \cdot Y_{\eta}$ ).

$$
\hat{J}_{\eta}\left(\hat{v}_{\hat{q}}\right):=\left\langle\hat{p}_{\hat{L}}\left(\hat{v}_{\hat{q}}\right), \hat{Y}_{\eta}(\hat{q})\right\rangle
$$

The tangent lift of a vector field $\hat{Z}$ on $\hat{Q}$ is the vector field $\hat{Z}^{T \hat{Q}}$ on $T \hat{Q}$ whose integral curves $t \mapsto \hat{v}(t)$ are velocities of integral curves $t \mapsto \hat{q}(t)$ of $\hat{Z}$, that is $\hat{v}(t)=\hat{Z}(q(t)) \in$ $T_{\hat{q}(t)} \hat{Q}$ (in coordinates, $Z^{T Q}=Z_{i} \partial_{q_{i}}+\dot{q}_{j} \frac{\partial Z_{i}}{\partial q_{j}} \partial_{\dot{q}_{i}}$ ). Clearly, $\hat{Y}_{\eta}^{T \hat{Q}}=\left.\frac{d}{d t} \hat{\Psi}_{\exp (t \eta)}^{T \hat{Q}}\right|_{t=0}$.

Consider now a nonholonomic mechanical system with affine constraints $(\hat{L}, \hat{Q}, \hat{M})$ and assume that $\hat{L}$ is invariant under $\hat{\Psi}^{T \hat{Q}}$, namely $\hat{L} \circ \hat{\Psi}_{g}^{T \hat{Q}}=\hat{L}$ for all $g \in G$. Then, we say that the function $\left.\hat{J}_{\eta}\right|_{\hat{M}}$ is the momentum of $(\hat{L}, \hat{Q}, \hat{M})$ generated by $\hat{Y}_{\eta}$.

Proposition 2. Assume that $\hat{L}$ is invariant under $\hat{\Psi}^{T \hat{Q}}$. Then a momentum is a first integral of $(\hat{L}, \hat{Q}, \hat{M})$ if and only if its generator is a section of $\hat{\mathcal{R}}^{\circ}$.

Proof. We may work in coordinates. A computation gives $\frac{d}{d t}\left(\left.J_{\eta}\right|_{M}\right)=\left.Y_{\eta}^{T Q}(L)\right|_{M}+$ $\left.R_{L, M} \cdot Y_{\eta}\right|_{M}$. The invariance of $L$ implies $Y_{\eta}^{T Q}(L)=0$. Thus, $\left.J_{\eta}\right|_{M}$ is a first integral if and only if, at each $q \in Q, Y_{\eta}$ annihilates all reaction forces $R_{L, M}(q, \dot{q})$ with $\dot{q} \in \mathcal{M}_{q}$, that is, $Y_{\eta}(q) \in \mathcal{R}_{q}^{\circ}$. 
4.2. Conservation of gauge momenta. It was an original idea of 4 that, for nonholonomic mechanical systems with linear constraints whose Lagrangian is invariant under a lifted action, certain conserved quantities that are not components of the momentum map may be viewed as linked to the group by a gauge-like mechanism. This situation extends to nonholonomic mechanical systems with affine constraints. For a general study of this topic in systems with linear constraints, and more information on the topic, including e.g. its relation to the so called 'momentum equation', see [15, 16, 19, 17].

Following the terminology of 16 we say that a vector field $\hat{Y}$ on $\hat{Q}$ is a gauge symmetry of $(\hat{L}, \hat{Q}, \hat{M})$ relative to the action $\hat{\Psi}$ if it is everywhere tangent to the orbits of $\hat{\Psi}$ and, moreover,

$$
\left.\hat{Y}^{T \hat{Q}}(\hat{L})\right|_{\hat{M}}=0
$$

This invariance condition of $\hat{L}$ is independent of the invariance of $\hat{L}$ under $\hat{\Psi}^{T \hat{Q}}$, even though it implies that $\hat{V}$ is $\hat{\Psi}$-invariant. The gauge momentum generated by a gauge symmetry $\hat{Y}$ is the function

$$
\hat{J}:=\left.\left\langle\hat{p}_{\hat{L}}, \hat{Y}\right\rangle\right|_{\hat{M}} .
$$

The gauge symmetry that generates a given gauge momentum needs not be unique.

Proposition 3. A gauge momentum is a first integral of $(\hat{L}, \hat{Q}, \hat{M})$ if and only if it is generated by a gauge symmetry which is a section of $\mathcal{R}^{\circ}$.

The proof goes just as that of Proposition 2.

The need of considering gauge momenta generated by gauge symmetries that are sections of $\mathcal{R}^{\circ}$, not only those generated by sections of $\mathcal{D}$, is demonstrated by the example in the following section. This is the heavy sphere that rolls inside a rotating vertical cylinder. This system has as a first integral that depends smoothly on the angular velocity of the cylinder and can be interpreted as a gauge momentum. Interestingly, when the cylinder is at rest this gauge momentum is generated by a gauge symmetry that is a section of $\mathcal{D}$; but as soon as the cylinder rotates, the generating gauge symmetry leaves $\mathcal{D}$ and becomes a section of $\mathcal{R}^{\circ}$.

4.3. Example. Consider a heavy sphere that rolls without sliding inside a cylinder that rotates uniformly about its vertical axis, namely, the system of section 3.2 with potential energy $V=g z$. The Lagrangian $\hat{L}$ and the configuration manifold $\hat{Q}$ are independent of $\Omega$, while the constraint manifold $\hat{M}$ depends on $\Omega$ through the vector field $\hat{\xi}$. We thus denote it by $\hat{M}_{\Omega}$.

It is classically known [29, 27] that, when $\Omega=0$, the system $\left(\hat{L}, \hat{Q}, \hat{M}_{0}\right)$ has the two first integrals $\hat{F}_{0}:=\left.\hat{F}\right|_{\hat{M}_{0}}$ and $\hat{K}_{0}:=\left.\hat{K}\right|_{\hat{M}_{0}}$, where

$$
\hat{F}=I \omega_{z}-a r \dot{\gamma}, \quad \hat{K}=a\left(\omega_{x} \cos \gamma+\omega_{y} \sin \gamma\right)-z \dot{\gamma}
$$

These two first integrals have been linked in [4] to the action $\hat{\Psi}$ of the group $G=$ $S^{1} \times \mathrm{SO}(3) \ni(\zeta, S)$ on $\hat{Q} \ni(z, \gamma, \mathcal{R})$ given by $\hat{\Psi}_{\zeta, S}(z, \gamma, \mathcal{R})=\left(z, \gamma+\zeta, S_{\zeta} \mathcal{R} S\right)$, where $S_{\zeta}$ is the matrix of the rotation by $\zeta$ about the third axis. This action emerges naturally in this problem because it leaves the Lagrangian (10) and the constraint (11) invariant. With reference to this action, $\hat{F}_{0}$ is a momentum generated by an infinitesimal generator that is a section of $\hat{\mathcal{D}}$ and $\hat{K}_{0}$ is a gauge momentum generated by a gauge symmetry which is a section of $\hat{\mathcal{D}}$, see 4 .

When $\Omega \neq 0$, the system $\left(\hat{L}, \hat{Q}, \hat{M}_{\Omega}\right)$ has the two first integrals

$$
\hat{F}_{\Omega}:=\left.\hat{F}\right|_{\hat{M}_{\Omega}}, \quad \hat{K}_{\Omega}:=\left.\hat{K}\right|_{\hat{M}_{\Omega}}
$$


which appear in 9]. (Reference 9 considers only the case $V=0$, but these two first integrals exist for any $V=V(z)$, see the remark below). We now show that, with reference to the considered action $\hat{\Psi}$, when $\Omega \neq 0$ the function $\hat{F}_{\Omega}$ is a momentum generated by an infinitesimal generator which is a section of $\hat{\mathcal{D}}$ and the function $\hat{K}_{\Omega}$ is a gauge momentum generated by a gauge symmetry which is a section of $\hat{\mathcal{R}}^{\circ}$, not of $\hat{\mathcal{D}}$. Therefore, at variance from the case $\Omega=0$, in order to link the first integral $\hat{K}_{\Omega}$ to the group action using the gauge mechanism, when $\Omega \neq 0$ it is necessary to take into account the role of the reaction forces.

To prove these assertions we pass to the local coordinates $(z, \gamma, \varphi, \psi, \theta)$ on $\hat{Q}$. The representatives $F$ of $\hat{F}$ and $K$ of $\hat{K}$ are obtained from (12) with $\omega_{x}=\dot{\theta} \cos \varphi+\dot{\psi} \sin \varphi \sin \theta$, $\omega_{y}=\dot{\theta} \sin \varphi-\dot{\psi} \cos \varphi \sin \theta, \omega_{z}=\dot{\varphi}+\dot{\psi} \cos \theta$. It follows from the analysis of section 3.2 that, in these coordinates,

$$
\begin{aligned}
& \mathcal{D}=\operatorname{span}_{\mathbb{R}}\left\{a \partial_{\gamma}-r \partial_{\varphi}, \partial_{\theta}-a \sin (\gamma-\varphi) \partial_{z}, \partial_{\psi}-a \cos (\gamma-\varphi) \sin \theta \partial_{z}-\cos \theta \partial_{\varphi}\right\} \\
& \mathcal{R}^{\circ}=\operatorname{span}_{\mathbb{R}}\left\{\partial_{\gamma}, \partial_{\varphi}, \partial_{\theta}-a \sin (\gamma-\varphi) \partial_{z}, \partial_{\psi}-a \cos (\gamma-\varphi) \sin \theta \partial_{z}-\cos \theta \partial_{\varphi}\right\}
\end{aligned}
$$

The tangent spaces to the group orbits are spanned by $\partial_{\gamma}$ and by three infinitesimal generators of the $\mathrm{SO}(3)$-action, e.g. the generators

$$
\begin{aligned}
& \eta_{x}=\sin \varphi\left(\partial_{\psi}-\cos \theta \partial_{\varphi}\right)+\cos \varphi \sin \theta \partial_{\theta}, \\
& \eta_{y}=\cos \varphi\left(\partial_{\psi}-\cos \theta \partial_{\varphi}\right)-\sin \theta \sin \varphi \partial_{\theta}, \\
& \eta_{z}=\partial_{\varphi}
\end{aligned}
$$

of the components $\omega_{x}, \omega_{y}, \omega_{z}$ of the $\mathrm{SO}(3)$-momentum map. The vector fields

$$
Y_{F}:=\eta_{z}-\frac{a}{r} \partial_{\gamma}, \quad Y_{K}:=\frac{a}{I \sin \theta}\left(\eta_{x} \cos \gamma-\eta_{y} \sin \gamma\right)-\frac{z}{r^{2}} \partial_{\gamma}
$$

are tangent to the group orbits. $Y_{F}$ is an infinitesimal generator of the $S^{1} \times \mathrm{SO}(3)$-action and is a section of $\mathcal{D}$. As such it generates a conserved momentum, that equals $F_{\Omega}$. $Y_{K}$ is instead a section of $\mathcal{R}^{\circ}$ and is (the local representative of) a gauge symmetry because

$$
Y^{T Q}(L)=\left(\dot{z}+a \omega_{x} \sin \gamma-a \omega_{y} \cos \gamma\right) \dot{\gamma}
$$

vanishes on $M_{\Omega}$, see (11). Thus, $Y_{K}$ generates a conserved gauge momentum, which equals $K_{\Omega}$.

If we use coordinates $(z, \gamma, \varphi, \psi, \theta, \dot{\varphi}, \dot{\psi}, \dot{\theta})$ on $\hat{M}_{\Omega}$, then

$$
F_{\Omega}=\left(I+a^{2}\right) \omega_{z}-a(a+r) \Omega, \quad K_{\Omega}=a \omega_{x} \cos \gamma+a \omega_{y} \sin \gamma+\frac{a}{r} z \omega_{z}-\frac{r+a}{r} \Omega z .
$$

It remains to prove that $K_{\Omega}$ is not generated by any gauge symmetry which is a section of $\mathcal{D}$. To this end, we make the following observations. We call generator of a gauge momentum any vector field $Z$-not necessarily a gauge symmetry - such that $\hat{J}=\left.\left\langle\hat{p}_{\hat{L}}, \hat{Z}\right\rangle\right|_{\hat{M}}$. Then, a gauge momentum has at most one generator that is a section of $\mathcal{D}$. We may prove this in coordinates. If $W$ and $Z$ are the representatives of two generators of a gauge momentum $\hat{J}$ then $\left.(W-Z) \cdot(A \dot{q}-b)\right|_{M}=0$. Equivalently, at each point $q,(W-Z) \cdot(A u+A \xi-b)=0$ for all $u \in \mathcal{D}_{q}$. Hence $Z$ and $W$ satisfy the two conditions

$$
(W-Z) \cdot A u=0 \forall u \in \mathcal{D}_{q}, \quad(W-Z) \cdot(A \xi-b)=0 .
$$

Since $A$ defines a metric, the first of these two conditions implies that, at each point $q$, $W-Z$ is orthogonal, in this metric, to $\mathcal{D}_{q}$. Hence all generators of a gauge momentum 
have the same component along $\mathcal{D}$. This argument shows that, moreover, if $Y$ is a generator of a gauge momentum, then its unique generator which is a section of $\mathcal{D}$, if it exists, is $\Pi_{A} Y$, where, at each point $q, \Pi_{A}$ is the $A$-orthogonal projector onto $\mathcal{D}_{q}$. Furthermore, $\Pi_{A} Y$ is a generator of the gauge momentum if and only if $\left(\Pi_{A} Y-Y\right)$. $(A \xi-b)=0$.

In our case $\Pi_{A} Y_{K}=-\frac{a^{2} z}{\left(a^{2}+I\right) r^{2}} \partial_{\gamma}+\left(\frac{a z}{\left(a^{2}+I\right) r}+\frac{a \cos \theta \sin (\gamma-\varphi)}{I \sin \theta}\right) \partial_{\varphi}-\frac{a \sin (\gamma-\varphi)}{I \sin \theta} \partial_{\psi}+$ $\frac{a}{I} \cos (\gamma-\varphi) \partial_{\theta}$ is not a generator of $K_{\Omega}$ since $\left(\Pi_{A} Y_{K}-Y_{K}\right) \cdot A \xi=\frac{I(a+r) \Omega z}{\left(a^{2}+I\right) r}$.

Remark. We have considered the case of constant gravity, with potential energy $V(z)=$ $g z$, in order to make a direct comparison with [4, that treats only this case. However, it is easy to verify that the two first integrals $F_{\Omega}$ and $K_{\Omega}$ exist, and retain their interpretations as (gauge) momenta, with the same generators, if the sphere is acted upon by any potential energy that depends only on $z$, that is, which is invariant under the considered action $\hat{\Psi}$. This is a 'Noether-like' property, called 'weak-Noetherianity' in [15]. This example thus shows that, in this respect, the case of nonholonomic systems with affine constraints differs from that of nonholonomic systems with linear constraints, where the only gauge momenta with such a weakly-Noetherian property are those that have a generator which is a section of $\mathcal{D}[17$.

\section{Conclusions}

At the core of Proposition 1 lies the balance equation of the energy (7). Together with the assumption of ideality of the constraints it implies that conservation of energy is equivalent to

$$
\langle\hat{R}(\hat{v}), \hat{\xi}(\hat{v})\rangle=0 \quad \forall v \in \hat{M}
$$

that is, to the fact that, in any constrained kinematical state, the reaction force $\hat{R}$ does not do any work on the nonhomogeneous term $\hat{\xi}$ of the constraint.

If the only knowledge assumed on the reaction forces is d'Alembert principle, namely that they annihilate the distribution $\hat{\mathcal{D}}$, then all can be deduced from equation (13) is that there is conservation of energy when $\hat{\xi}$ is a section of $\hat{\mathcal{D}}$, that is, when the constraint is linear. This point of view is quite widespread: the very use of expressions such as "undetermined multipliers" or "unknown [reaction] forces" indicates a perception of the reaction forces as intrinsically unknown objects.

The novelty of our approach is that we exploit the fact that, for a given system, the reaction forces are known functions of the kinematic states. This allows to identify the cases in which the energy is conserved even if the constraint is genuinely affine.

Very similar considerations can be made concerning the conservation of momenta and gauge momenta.

The general message we convey, besides the specific information about the conservation of energy and (gauge) momenta in nonholonomic mechanical systems with affine constraints, is that the reaction forces should be taken into a clearer account in theoretical studies of these systems. Particularly when analyzing differences from holonomic systems (Noether theorem, conservation of energy, hamiltonianization, perhaps invariant measures) it might be important to exploit the presence of the reaction forces in the equations of motion. From this point of view, advancement in the comprehension of nonholonomic mechanical systems is not a purely geometric matter and the comprehension of some dynamical aspects might pass through a better understanding - and perhaps attempts of classification - of the reaction forces. 


\section{Appendix: The equations of motion and the reaction forces}

We derive here the expression of the reaction forces as functions of the kinematic states of the system for a nonholonomic system with affine constraints.

Proposition 4. In the hypotheses and with the notation of section 2.1:

1. For any $\bar{v}_{0} \in \hat{M}$ there exist unique curves $\mathbb{R} \ni t \mapsto\left(\hat{v}_{t}, \hat{R}_{t}\right) \in \hat{M} \times \hat{\mathcal{D}}^{\circ}$ such that $\hat{v}_{0}=\bar{v}_{0}$ and

$$
[\hat{L}]\left(\hat{v}_{t}\right)=\hat{R}_{t} \quad \forall t .
$$

2. There exists a function $\hat{R}_{\hat{L}, \hat{M}}: \hat{M} \rightarrow \hat{\mathcal{D}}^{\circ}$ such that, for all $\bar{v}_{0}, \hat{R}_{t}=\hat{R}_{\hat{L}, \hat{M}}\left(\hat{v}_{t}\right) \forall t$.

3. In any system of bundle coordinates $(q, \dot{q})$ in $T \hat{Q}$, if $M$ is described as $S(q) \dot{q}+s(q)=$ 0 , then the representative $R_{L, M}$ of $\hat{R}_{\hat{L}, \hat{M}}$ is the restriction to $M$ of the function (5), (6).

Proof. We first work in coordinates and then globalize the result. The matrix $A=$ $\frac{\partial^{2} L}{\partial \dot{q} \partial \dot{q}}=\frac{\partial^{2} L_{2}}{\partial \dot{q} \partial \dot{q}}$ is nonsingular and independent of the velocities.

Assume $t \mapsto\left(q_{t}, \dot{q}_{t}, R_{t}\right)$ is the local representative of a curve in $M \times \mathcal{D}^{\circ}$ that satisfies (14). Then, for all $t$,

$$
S\left(q_{t}\right) \dot{q}_{t}+s\left(q_{t}\right)=0
$$

and $R_{t} \in$ range $S\left(q_{t}\right)^{T}$. Thus, there exists a curve $t \mapsto \lambda_{t} \in \mathbb{R}^{k}$ (the Lagrange multiplier) such that $R_{t}=S\left(q_{t}\right)^{T} \lambda_{t}$. Since $[L]=A(q) \ddot{q}+\ell(q, \dot{q})$, equation (14) is

$$
A\left(q_{t}\right) \ddot{q}_{t}+\ell\left(q_{t}, \dot{q}_{t}\right)=S\left(q_{t}\right)^{T} \lambda_{t}
$$

and gives $\ddot{q}_{t}=A\left(q_{t}\right)^{-1}\left[S\left(q_{t}\right)^{T} \lambda_{t}-\ell\left(q_{t}, \dot{q}_{t}\right)\right]$. Inserting this expression into $S\left(q_{t}\right) \ddot{q}_{t}+$ $\sigma\left(q_{t}, \dot{q}_{t}\right)=0$, that follows from (15), gives

$$
S\left(q_{t}\right) A\left(q_{t}\right)^{-1}\left[S\left(q_{t}\right)^{T} \lambda_{t}-\ell\left(q_{t}, \dot{q}_{t}\right)\right]+\sigma\left(q_{t}, \dot{q}_{t}\right)=0 .
$$

This equation can be solved for $\lambda_{t}$ because the matrix $S A^{-1} S^{T}$ is invertible and gives

$$
\lambda_{t}=\left[S\left(q_{t}\right) A\left(q_{t}\right)^{-1} S\left(q_{t}\right)^{T}\right]^{-1}\left[S\left(q_{t}\right) A\left(q_{t}\right)^{-1} \ell\left(q_{t}, \dot{q}_{t}\right)-\sigma\left(q_{t}, \dot{q}_{t}\right)\right]
$$

or $R_{t}=R_{L, M}\left(q_{t}, \dot{q}_{t}\right)$ with $R_{L, M}$ as stated. Together with the independence of $R_{L, M}$ of the choice of $S$ and $s$, see the remark at the end of section 2.2, this proves that if $\hat{R}_{\hat{L}, \hat{M}}$ exists, then its local representative is $R_{L, M}$ and hence the uniqueness of $t \mapsto\left(q_{t}, \dot{q}_{t}, R_{t}\right) \in$ $M \times \mathcal{D}^{\circ}$, as in item 1 .

Consider now the equation

$$
A \ddot{q}+\ell=S^{T}\left(S A^{-1} S^{T}\right)^{-1}\left(S A^{-1} \ell-\sigma\right)
$$

in $\mathbb{R}^{n}$. Let $t \mapsto q_{t}$ be its unique solution with initial datum $\left(q_{0}, \dot{q}_{0}\right) \in M$. Define $t \mapsto \lambda_{t}$ as in (16). With this choice of $\lambda_{t}$, the curve $t \mapsto q_{t}$ satisfies $S \ddot{q}_{t}+\sigma\left(q_{t}, \dot{q}_{t}\right)=0$, or $\frac{d}{d t}\left[S\left(q_{t}\right) \dot{q}_{t}+s\left(q_{t}\right)\right]=0$. Hence $\left(q_{t}, \dot{q}_{t}\right) \in M$ for all $t$ and satisfies equation (14) with $R_{t}=S\left(q_{t}\right)^{T} \lambda_{t} \in \mathcal{D}_{q_{t}}^{\circ}$. By d'Alembert principle, such an $R_{t}$ is the representative of a reaction force that the nonholonomic constraint can exert. chart.

This proves a local version of items 1 . and 2., which hold true within each coordinate

We now globalize these results. To this end we verify that the local representatives of equation (14), with reaction forces that have the expression (5), match in the intersection 
of different chart domains. Let $\tilde{q} \mapsto q=\mathcal{C}(\tilde{q})$ be a change of coordinates in $Q$. The local representatives of the affine subbundle $M$ in the new coordinates is still given by an equation of the form $\tilde{S}(\tilde{q}) \dot{\tilde{q}}+\tilde{s}(\tilde{q})=0$ and a simple computation shows that

$$
\tilde{S}=\tilde{P}[S \circ \mathcal{C}] \mathcal{C}^{\prime}, \quad \tilde{s}=\tilde{P}[s \circ \mathcal{C}]
$$

where $\mathcal{C}^{\prime}$ is the Jacobian matrix of $\mathcal{C}$ and $\tilde{P}=\tilde{P}(\tilde{q})$ is a $k \times k$ nonsingular matrix. The local representatives $\tilde{L}$ of the Lagrangian in the new coordinates is $\tilde{L}(\tilde{q}, \dot{\tilde{q}})=L\left(\mathcal{C}(\tilde{q}), \mathcal{C}^{\prime}(\tilde{q}) \dot{\tilde{q}}\right)$ and the matrix $\tilde{A}:=\frac{\partial^{2} \tilde{L}}{\partial \tilde{q} \partial \tilde{q}}$ is given by

$$
\tilde{A}=\mathcal{C}^{\prime T}[A \circ \mathcal{C}] \mathcal{C}^{\prime}
$$

In order to prove the statement it suffices to show that, if $t \mapsto q_{t}$ is a solution of

$$
\frac{d}{d t} \frac{\partial L}{\partial \dot{q}}-\frac{\partial L}{\partial q}=S^{T}\left(S A^{-1} S^{T}\right)^{-1}\left(S A^{-1} \ell-\sigma\right),
$$

then $t \mapsto \tilde{q}_{t}=\mathcal{C}^{-1}\left(q_{t}\right)$ is a solution of

$$
\frac{d}{d t} \frac{\partial \tilde{L}}{\partial \dot{\tilde{q}}}-\frac{\partial L}{\partial \tilde{q}}=\tilde{S}^{T}\left(\tilde{S} \tilde{A}^{-1} \tilde{S}^{T}\right)^{-1}\left(\tilde{S} \tilde{A}^{-1} \tilde{\ell}-\tilde{\sigma}\right)
$$

with $\tilde{\ell}$ and $\tilde{\sigma}$ defined as $\ell$ and $\sigma$ in (6), but in terms of $\tilde{L}, \tilde{S}$ and $\tilde{s}$. It is well known from Lagrangian mechanics that

$$
\frac{d}{d t} \frac{\partial \tilde{L}}{\partial \dot{\tilde{q}}}-\frac{\partial \tilde{L}}{\partial \tilde{q}}=\mathcal{C}^{\prime T}\left[\left(\frac{d}{d t} \frac{\partial L}{\partial \dot{q}}-\frac{\partial L}{\partial q}\right) \circ \mathcal{C}\right] .
$$

Elementary computations show that

$$
\begin{aligned}
& \tilde{\ell}_{i}=\left(\mathcal{C}^{\prime T} \ell\right)_{i}+\left(\mathcal{C}^{\prime T} A\right)_{i j}\left[\dot{\tilde{q}} \cdot \mathcal{C}_{j}^{\prime \prime} \dot{\tilde{q}}\right] \\
& \tilde{\sigma}_{a}=(\tilde{P} \sigma)_{a}+(\tilde{P} S)_{a j}\left[\dot{\tilde{q}} \cdot \mathcal{C}_{j}^{\prime \prime} \dot{\tilde{q}}\right]
\end{aligned}
$$

where $\mathcal{C}_{j}^{\prime \prime}$ is the Hessian matrix of the $j$-th component $\mathcal{C}_{j}$ of $\mathcal{C}$ and with the convention, used below as well, that $\ell, A, \sigma$ and $S$ are composed with $\mathcal{C}$. Since $\tilde{S} \tilde{A}^{-1}=\tilde{P} S A^{-1} \mathcal{C}^{\prime-T}$, this implies $\tilde{S} \tilde{A}^{-1} \tilde{\ell}-\tilde{\sigma}=\tilde{P}\left[S A^{-1} \ell-\sigma\right]$ so that

$$
\tilde{S}^{T}\left(\tilde{S} \tilde{A}^{-1} \tilde{S}^{T}\right)^{-1}\left[\tilde{S} \tilde{A}^{-1} \tilde{\ell}-\tilde{\sigma}\right]=\mathcal{C}^{\prime T}\left[\left(S^{T}\left(S A^{-1} S^{T}\right)^{-1}\left[S A^{-1} \ell+\sigma\right]\right) \circ \mathcal{C}\right]
$$

This completes the proof.

\section{References}

[1] R. Abraham and J. E. Marsden, Foundations of Mechanics (Benjamin, Reading 1978).

[2] C. Agostinelli, Nuova forma sintetica delle equazioni del moto di un sistema anolonomo ed esistenza di un integrale lineare nelle velocità. Boll. Un. Mat. Ital. 11 (1956), 1-9.

[3] V.I. Arnold, Mathematical Methods of Classical Mechanics. Graduate Texts in Mathematics 60 (Springer-Verlag, New York, 1989).

[4] L. Bates, H. Graumann and C. MacDonnell, Examples of gauge conservation laws in nonholonomic systems. Rep. Math. Phys. 37 (1996), 295-308. 
[5] L.M. Bates and J.M Nester, On D'Alembert's principle. Commun. Math. 19 (2011), 57-72.

[6] L. Bates and J. Śniatycki, Nonholonomic reduction. Rep. Math. Phys. 32 (1993), 99-115.

[7] S. Benenti, A 'user-friendly' approach to the dynamical equations of non-holonomic systems. SIGMA Symmetry Integrability Geom. Methods Appl. 3 (2007), Paper 036, 33 pp.

[8] A.M. Bloch, P.S. Krishnaprasad, J.E. Marsden and R.M. Murray, Nonholonomic mechanical systems with symmetry. Arch. Rational Mech. Anal. 136 (1996), 21-99.

[9] A.V. Borisov, I.S. Mamaev and A.A. Kilin, The rolling motion of a ball on a surface. New integrals and hierarchy of dynamics. Regul. Chaotic Dyn. 7 (2002), 201-219.

[10] A.V. Borisov and I.S. Mamaev, Conservation Laws, Hierarchy of Dynamics and Explicit Integration of Nonholonomic Systems. Regul. Chaotic Dyn. 13 (2008), 443-490.

[11] F. Cantrijn, M. de Leon, D. Martin de Diego and J.C. Marrero, Reduction of nonholonomic mechanical systems with symmetries. Rep. Math. Phys. 42 (1998), 25-45.

[12] J. Cortés Monforte, Geometric, Control and Numerical Aspects of Nonholonomic Systems. Lecture Notes in Math. 1793 (Springer-Verlag, Berlin, 2002).

[13] M. Crampin and T. Mestdag, The Cartan form for constrained Lagrangian systems and the nonholonomic Noether theorem. Int. J. Geom. Methods. Mod. Phys. 8 (2011), 897-923.

[14] R. Cushman, J.J. Duistermaat and J. Śniatycki, Geometry of Nonholonomically Constrained Systems. Advanced Series in Nonlinear Dynamics 26 (World Scientific, Singapore, 2010)

[15] F. Fassò, A. Giacobbe and N. Sansonetto, Gauge conservation laws and the momentum equation in nonholonomics mechanics. Rep. Math. Phys. 62 (2008), 345-367.

[16] F. Fassò, A. Giacobbe and N. Sansonetto, On the number of weakly Noetherian constants of motion of nonholonomic systems. J. Geom. Mech. 1 (2009), 389-416.

[17] F. Fassò, A. Giacobbe and N. Sansonetto, Linear weakly Noetherian constants of motion are horizontal gauge momenta. J. Geom. Mech. 4, 2 (2012) 129-136.

[18] F. Fassò, A. Ramos and N. Sansonetto, The reaction-annihilator distribution and the nonholonomic Noether theorem for lifted actions. Regul. Chaotic Dyn. 12 (2007), 449-458.

[19] F. Fassò and N. Sansonetto, An elemental overview of the nonholonomic Noether theorem. Int. J. Geom. Methods Mod. Phys. 6 (2009), 1343-1355.

[20] F. Fassò and N. Sansonetto, Conservation of 'moving' energy in nonholonomic systems with affine constraints and integrability of spheres on rotating surfaces. Preprint (2015). http://arxiv.org/abs/1503.06661

[21] G. Gorni and G. Zampieri, Time reversibility and energy conservation for Lagrangian systems with nonlinear nonholonomic constraints. Reports on Mathematical Physics 45 (2000), 217-227.

[22] M. Jotz and T. Ratiu, Dirac structures, nonholonomic systems and reduction. Rep. Math. Phys. 69 (2012), 5-56.

[23] M.H. Kobayashi and W.O. Oliva, A note on the conservation of energy and volume in the setting of nonholonomic mechanical systems. Qual. Theory Dyn. Syst. 5, 4 (2004) 247-259.

[24] C.-M. Marle, Reduction of constrained mechanical systems and stability of relative equilibria. Comm. Math. Phys. 174, 2 (1995) 295-318.

[25] C.-M. Marle, On symmetries and constants of motion in Hamiltonian systems with nonholonomic constraints. In Classical and Quantum Integrability (Warsaw, 2001), 223-242, Banach Center Publ. 59 (Polish Acad. Sci. Warsaw, 2003).

[26] E. Massa and E. Pagani, Classical dynamics of nonholonomic systems: a geometric approach. Ann. Inst. H. Poincaré Phys. Théor. 55 (1991), 511-544. 
[27] J.I. Neimark and N.A. Fufaev, Dynamics of Nonholonomic Systems. Translations of Mathematical Monographs 33 (AMS, Providence, 1972).

[28] L.A. Pars, A Treatise on Analytical Dynamics. (Heinemann, London 1965).

[29] E.J. Routh, Treatise on the Dynamics of a System of Rigid Bodies (Advanced Part). (Dover, New York, 1955).

[30] J. Śniatycki, Nonholonomic Noether theorem and reduction of symmetries. Rep. Math. Phys. 42 (1998), 5-23.

[31] G. Terra and M.H. Kobayashi, On Classical Mechanics with Nonlinear Constraints. J. Geom. Phys. 49, 3-4 (2003) 385-417. 